\title{
Relativistic two-center continuum
}

\author{
Karl-Heinz Wietschorke \\ Hoechst Aktiengesellschaft, Technische Leitsysteme, Frankfurt/Main, Federal Republic of Germany \\ Paul Schlüter \\ Siemens Aktiengesellschaft, Zentralbereich Forschung und Technik, München, Federal Republic of Germany \\ Gerhard Soff \\ Gesellschaft für Schwerionenforschung, D-6100 Darmstadt, Federal Republic of Germany \\ Klaus Rumrich and Walter Greiner \\ Institut für Theoretische Physik der Universität Frankfurt, Federal Republic of Germany \\ (Received 12 January 1987)
}

\begin{abstract}
A method is presented to define unique continuum states for the two-center Dirac Hamiltonian. In the spherical limit these states become the familiar angular-momentum eigenstates of the radial Coulomb potential. The different states for a fixed total energy $|E|>m$ may be distinguished by considering the asymptotic spin-angular distribution of states with unique scattering phases. The first numerical solutions of the two-center Dirac equation for continuum states are presented.
\end{abstract}

One important problem in a complete discussion of the spectrum of the two-center Dirac equation is the correct treatment of the continuum. There is considerable interest in these questions because the effects of electronic excitation and pair creation in collisions of very heavy ions generally are based on the quasimolecular model, i.e., on an expansion of the wave function in terms of the adiabatic solutions of the two-center Dirac equation. ${ }^{1} \mathrm{Up}$ to now the Dirac equation in the continuum has been solved in the so-called monopole approximation. ${ }^{2}$ It cannot be excluded that typical molecular effects are important for the excitation processes of interest. In particular, the typical appearance of a resonance in the lower Dirac continuum $(E<-m)$ for small two-center distances and overcritical united nuclei charges $\left(Z_{1}+Z_{2}>173\right)$ should be investigated. $^{3}$ Here we want to examine to what extent the probability density distribution differs from an isotropic shape obtained in the monopole approximation. ${ }^{4}$ Nonrelativistic calculations solving the two-center Schrödinger equation are not useful for our purpose, not even qualitatively. The stationary solutions of the Schrödinger equation can be separated into three factors using prolate elliptical coordinates which depend on the "radial" and the two "angle" coordinates. In contrast, the two-center Dirac Hamiltonian can be reduced only to a partial differential operator in at least two dimensions. The set of quantum numbers is also reduced by one in the relativistic case. The consequences can be observed in the correlation diagrams of the bound states, which were first determined by Müller et $a l .^{4,5}$ As an important difference, avoided level crossings are observed between states which in the nonrelativistic theory are allowed to cross due to the presence of an additional quantum number.

To find eigenstates in the continuous part of the spec- trum $(|E|>m)$ a criterion to classify the wave functions of the continuum has been lacking up to now. We will show that it is possible to define states in a unique manner by requiring asymptotically a well-defined scattering phase. To obtain regular wave functions fulfilling this criterion, we have to allow an asymptotic behavior of the outcoming stable spin-angular distribution far away from the potential centers which need not be correlated to a good angular momentum. One can verify the orthogonality also for such a set of continuum states with a fixed total energy $|E|>m$. We use the continuum wave functions, normalized to a $\delta$ function on the energy scale, to calculate coupling matrix elements with bound states for superheavy systems and compare them with results from the monopole approximation.

To become familiar with the possible continuum solutions of the two-center Dirac equation, an asymptotic investigation for large distances from the potential centers is useful. For convenience we will employ spherical coordinates for the following general considerations. Using the common standard representation, one finds that the upper and the lower spinor are connected by the linear matrix operator $\sigma_{r}=\mathbf{e}_{r} \cdot \boldsymbol{\sigma}$ for large distances $r$. But we are not restricted to special spin-angular distributions such as the eigenstates $\chi_{\kappa}^{\mu}(\vartheta, \phi)$ of the spherical $\hat{\boldsymbol{\kappa}}$ operator because asymptotically this operator has no more influence on the wave function. Nevertheless, we will check whether it is possible to retain an asymptotic behavior of the continuum states with good total angular momentum in the twocenter problem.

A solution of the two-center Dirac equation, with a unique scattering phase and an asymptotic behavior corresponding to a good total angular-momentum quantum number $\kappa$, reads for large $r$ 


$$
\left.\psi_{\kappa}^{\mu}(r, \vartheta, \phi)\right|_{r \rightarrow \infty}=\frac{1}{\sqrt{\pi p}} \frac{1}{r}\left[\begin{array}{cc}
\sqrt{|E+m|} \cos \left(p r+\delta_{\kappa}\right) \chi_{\kappa}^{\mu}(\vartheta, \phi) \\
i \operatorname{sgn}(E) & \sqrt{|E-m|} \sin \left(p r+\delta_{\kappa}\right) \sigma_{r} \chi_{\kappa}^{\mu}(\vartheta, \phi)
\end{array}\right) .
$$

We employ the notation of Rose. ${ }^{6}$ Due to the higher multipole moments of the potential, we get an increasing admixture of different spin-angular eigenfunctions $\chi_{\kappa}^{\mu}$, if we continue the wave function $\psi_{\kappa}^{\mu}$ to smaller $r$. We have to require that the part of the total wave function in an expansion in spherical $\kappa$ eigenstates which is irregular at the origin vanishes. Together with the freedom to determine the phase $\delta_{\kappa}$, this leads to the following conditions:

$$
\cos \delta_{\kappa} b_{\kappa \kappa^{\prime}}+\sin \delta_{\kappa} d_{\kappa \kappa^{\prime}}=0 \text { for all } \kappa^{\prime}
$$

Here $b_{\kappa \kappa^{\prime}}$ and $d_{\kappa \kappa^{\prime}}$ are the coefficients for those parts $\sim \chi_{\kappa^{\prime}}^{\mu}$ of the wave function which are divergent at the origin. Generally it will be impossible to fulfill all these equations with one phase $\delta_{\kappa}$. Only in the spherical limit in which (2) reduces to only one equation for every continuum state $\left(b_{\kappa \kappa^{\prime}}=d_{\kappa \kappa^{\prime}}=0\right.$ for $\left.\kappa^{\prime} \neq \kappa\right)$ can we introduce a continuum basis in this way.

Thus it becomes necessary to generalize (1) to get a realizable classification method. A modification of only the spin-angular distributions seems to be the practicable way. Alternatives are always connected with a nonunique (i.e., $r$ dependent) phase which would be unphysical if we consider the continuum states as scattering channels. It is easy to show that the degrees of freedom given by the coefficients of a possible expansion in the $\chi_{\kappa}^{\mu}(\vartheta, \phi)$ basis of a new "free" spin-angular distribution $\Theta_{\mu}(\vartheta, \phi)$ may be used to find a regular continuum solution with one welldefined scattering phase $\delta$. Because of the conservation of the $z$ component of the total angular momentum, $\Theta_{\mu}(\vartheta, \phi)$ has to be an eigenfunction of $J_{z}$ with the eigenvalue $\mu$. This means for an expansion of $\Theta_{\mu}(\vartheta, \phi)$ in the complete set $\left\{\chi_{\kappa}^{\mu}\right\}$ a limitation to a fixed quantum number $\mu$. The expansion of $\Theta_{\mu}$ in terms of $\chi_{\kappa}^{\mu}$ in general is $r$ dependent, but since the higher multipole components of the potential decrease very fast in the limit $r \rightarrow \infty$, only the monopole contributes in this limit. The monopole part is spherically symmetric, thus it does not change the angular distribution and $\Theta_{\mu}$ approaches an asymptotic value. With the normalization in the angular coordinates

$$
\left.\iint d \vartheta d \phi \sin \vartheta \Theta_{\mu}^{\dagger} \Theta_{\mu}\right|_{r \rightarrow \infty}=1,
$$

we are able to normalize the generalized continuum wave function in analogy to the spherical case with the asymptotic behavior

$$
\left.\psi_{\mu}(r, \vartheta, \phi)\right|_{r \rightarrow \infty}=(\pi p)^{-1 / 2} \frac{1}{r}\left[\begin{array}{c}
+\sqrt{|E+m|} \cos (p r+\delta) \Theta_{\mu}(\vartheta, \phi) \\
i \operatorname{sgn}(E) \sqrt{|E-m|} \sin (p r+\delta) \sigma_{r} \Theta_{\mu}(\vartheta, \phi)
\end{array}\right)
$$

In the following we will discuss the question of orthogonality between two continuum states. We consider two wave functions with energies $E$ and $E^{\prime}$ :

$$
\Psi=\psi(\mathbf{r}) e^{-i E t}, \quad \Psi^{\prime}=\psi^{\prime}(\mathbf{r}) e^{-i E^{\prime} t},
$$

and introduce the transition current $\bar{\Psi}^{\prime} \gamma^{\mu} \Psi$ fulfilling the continuity equation

$$
\operatorname{div}\left(\Psi^{\prime \dagger} \alpha \Psi\right)+\frac{\partial}{\partial t}\left(\Psi^{\prime \dagger} \Psi\right)=0
$$

We have a stationary system and so this equation is equivalent to

$$
\operatorname{div}\left(\psi^{\prime \dagger} \boldsymbol{\alpha} \psi\right)+i\left(E^{\prime}-E\right) \psi^{\prime \dagger} \psi=0 .
$$

Integrating this expression over a sphere with the radius $R$, we can use the Gaussian law and get

$$
\begin{aligned}
\iint d \vartheta & \left.d \phi R^{2} \sin \vartheta \psi^{\prime} \alpha_{r} \psi\right|_{r=R} \\
& =i\left(E-E^{\prime}\right) \int^{R} d r \iint d \vartheta d \phi r^{2} \sin \vartheta \psi^{\prime \dagger} \psi .
\end{aligned}
$$

The left-hand side has to vanish for $E \rightarrow E^{\prime}$ because the integral on the right is finite for regular wave functions. So (7) becomes

$$
\begin{aligned}
\left(E^{2}-m^{2}\right)^{1 / 2} & \sin \left(\delta-\delta^{\prime}\right) \\
& \times \iint d \vartheta d \phi \sin \vartheta \Theta_{\mu}^{\prime}(\vartheta, \phi) \Theta_{\mu}(\vartheta, \phi)=0
\end{aligned}
$$

after inserting (4), which is valid for values of $R$ large compared with the two-center distance. Trivially (9) is fulfilled for waves with the same phases $\delta=\delta^{\prime}$. Otherwise $\left(\delta \neq \delta^{\prime}\right)$ the angular wave functions $\Theta_{\mu}$ and $\Theta_{\mu^{\prime}}$ have to be orthogonal. In conclusion we see that the orthogonality of the functions $\Theta_{\mu}$ for different phases $\delta$ follows from the vanishing transition current between two stationary states with the same total energy. The continuum states for different energies are in the same sense orthogonal to each other as they are in the spherical case. A proof is based mainly on the questions of symmetry and self-adjointness of the Dirac Hamiltonian. ${ }^{7}$

We solve the two-center Dirac equation using an expansion in the orthogonal spin-angular distributions $\chi_{\kappa}^{\mu}(\vartheta, \phi)$ with good total angular quantum numbers $\kappa .{ }^{8}$ In this way we can reduce the partial differential equation to a coupled system of ordinary differential equations in the 


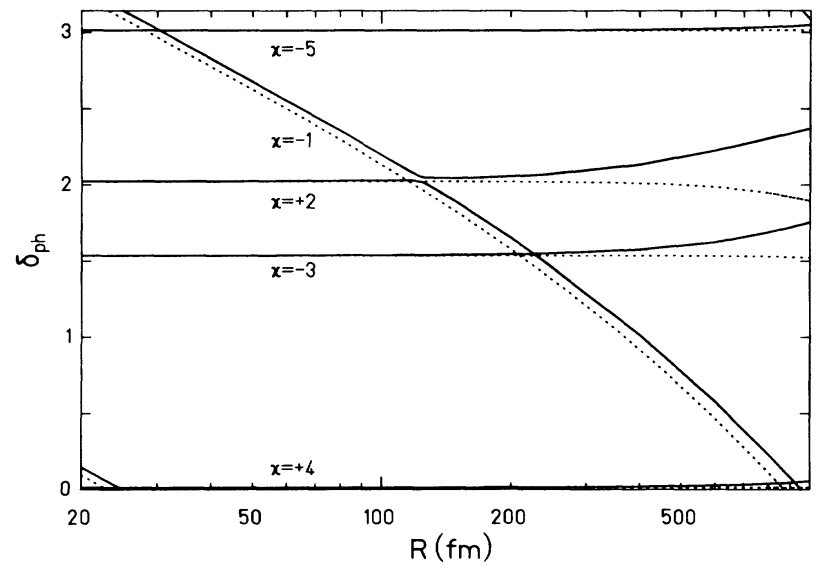

FIG. 1. The physical scattering phases as a function of the two-center distance of five even continuum states (solid curves) for a system of two extended lead nuclei, a total energy $E=+1.1 \mathrm{~m}$, and the magnetic quantum number $\mu=\frac{1}{2}$. The corresponding phases of five continuum states $(\kappa=-1,+2,-3,+4,-5)$ for the same system and quantum numbers resulting from the monopole approximation are depicted by the dotted curves.

radial coordinate. For the integration in this last variable we apply the computer code DE of Shampine and Gordon $^{9}$ which guarantees an accuracy better than $10^{-5}$ for the wave function in the chosen approximation.

In a discussion of our numerical results we shall start with the "phase correlation diagram" in Fig. 1. For the

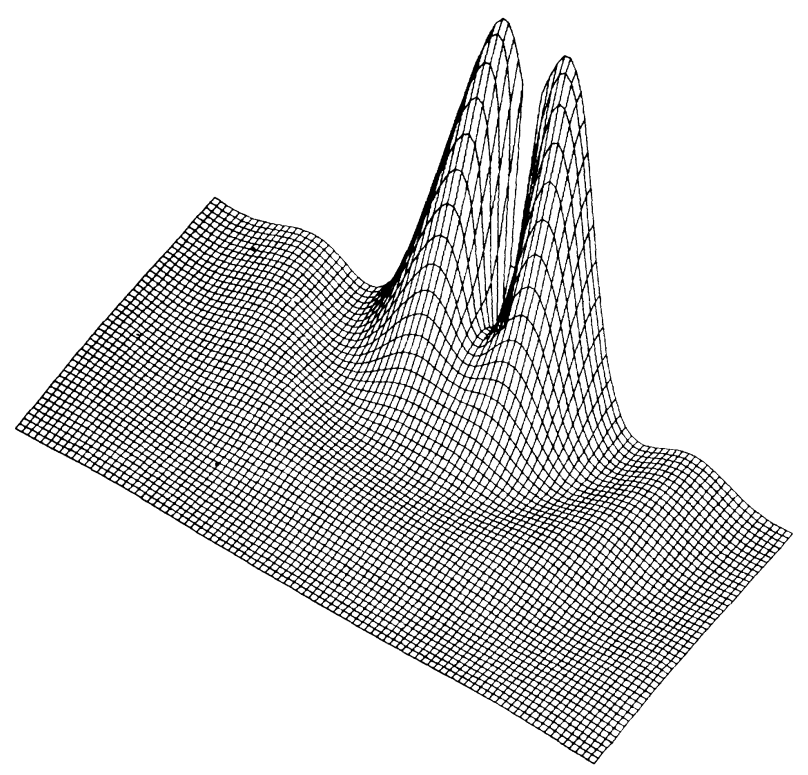

FIG. 2. The expectation probability density of a continuum " $d$ wave" (similar to $\kappa=+2, \mu=\frac{1}{2}$ ) for two extended lead nuclei at a total energy $E=+1.1 \mathrm{~m}$ is shown in an isometric representation. The two-center distance is $220 \mathrm{fm}$. The shown rectangular in the $(\rho, z)$ plane has the range $3750 \times 7500\left[\mathrm{fm}^{2}\right]$. symmetric system of two extended lead nuclei $\left(Z_{1}+Z_{2}=164\right)$ we have calculated the scattering phases of five even continuum states for the energy $E=+1.1 \mathrm{~m}$ and the magnetic quantum number $\mu=\frac{1}{2}$. We have chosen those states which possess spin-angular wave functions similar to the eigenstates of the spherical problem with the lowest "even" total angular-momentum quantum numbers $(\kappa=-1,+2,-3,+4,-5)$. In a comparison with the results for the same system and energy but within the monopole approximation (dotted curves), we immediately recognize one difference. As in the energy correlation diagrams of the discrete spectrum, it seems to be forbidden that two states coincide in all quantum numbers $\{E, \mu, \pi, \delta\}$. Besides this phenomenon, the agreement between full and dotted curves is fairly good. In the investigated region of the two-center distance we see no striking difference to the results of the monopole approximation if we disregard the characteristic mixing effects of the wave functions near the forbidden crossings.

The appearance of the density distribution of the continuum wave functions changes not fundamentally as a function of the two-center distance. We have added an isometric plot (Fig. 2) of a " $d$-wave" (correlating with $\kappa=+2$ in the spherical limit) density of the same system with the quantum number $\mu=\frac{1}{2}$ and a total energy $E=+1.1 \mathrm{~m}$ at a two-center distance of $220 \mathrm{fm}$ for illus-

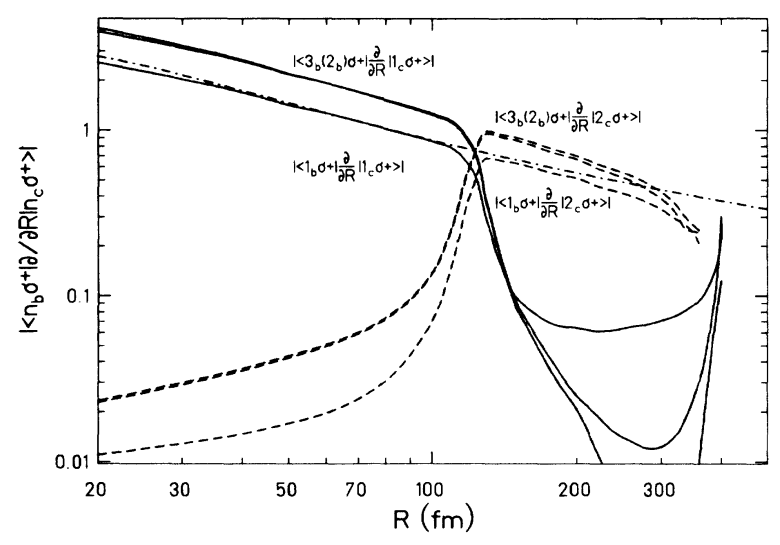

FIG. 3. The radial coupling matrix elements in natural units between the three strongest bound even states and two even continuum states with a total energy $E=+1.1 \mathrm{~m}$ and the quantum number $\mu=\frac{1}{2}$ are shown as a function of the two-center distance for the system of two extended lead nuclei. The continuum and the bound wave function are denoted by the subscripts $c$ and $b$. The sign + indicates the current parity. In the considered two-center region the three bound states are comparable to the following spherical states in the monopole approximation $1_{b} \sigma+: 1 s \frac{1}{2}, 2_{b} \sigma+: 2 s \frac{1}{2}$, and $3_{b} \sigma+: 3 s \frac{1}{2}$. The two continuum states are dominated by their spherical components with the angular quantum numbers $\kappa=-1\left(1_{c} \sigma+\right)$ and $\kappa=+2\left(2_{c} \sigma+\right)$. The dash-dot curve is the monopole approximation matrix element $\left\langle 1 s \frac{1}{2}|\partial / \partial R| E=1.1, \kappa=-1\right\rangle$. 
tration. It is nearly the same shape as in the spherical limit although the position of the two potential centers coincides now approximately with the position of the first bumps in the density. There are also small deviations in the radial coupling, cf. Fig. 3, from bound states into the upper continuum as compared to the results of calcula- tions within the monopole approximation. Naturally one can also observe here the consequences of the forbidden crossings.

We thank Dr. J. Reinhardt for fruitful discussions and a careful reading of the manuscript.
${ }^{1}$ W. Greiner, B. Müller, and J. Rafelski, Quantum Electrodynamics of Strong Fields (Springer Verlag, Berlin, 1985).

${ }^{2}$ G. Soff, W. Betz, J. Reinhardt, and J. Rafelski, Phys. Scr. 17, 417 (1978).

${ }^{3}$ B. Müller, J. Rafelski, and W. Greiner, Z. Phys. 257, 62 (1972); 257, 183 (1972); Nuovo Cimento 18A, 551 (1973).

${ }^{4} \mathrm{G}$. Soff, W. Greiner, W. Betz, and B. Müller, Phys. Rev. A 20 , 169 (1979); G. Soff, J. Reinhardt, and W. Greiner, Phys. Lett. 83A, 158 (1981).

${ }^{5}$ B. Müller, J. Rafelski, and W. Greiner, Phys. Lett. 47B, 5
(1973); B. Müller and W. Greiner, Z. Naturforsch, Teil A 31 1 (1976).

${ }^{6}$ E. M. Rose, Relativistic Electron Theory (Wiley, New York, 1961).

${ }^{7}$ R. D. Richtmyer, Principles of Advanced Mathematical Physics (Springer-Verlag, New York, 1978).

${ }^{8}$ J. Rafelski and B. Müller, Phys. Rev. Lett. 36, 517 (1976).

${ }^{9}$ L. F. Shampine and M. K. Gordon, Computer Solutions of Ordinary Differential Equations: The Initial Value Problem (Freeman, San Francisco, 1975). 